\title{
KINETIC AND SPECTROSCOPIC STUDIES OF TRANSIENT SPECIES PREPARED BY IRMPD
}

\author{
F. CASTAÑO, $\dagger$ A. ORTIZ DE ZÁRATE, J. A. FERNÁNDEZ \\ and M. N. SÁNCHEZ RAYO \\ Departmento de Química Física. Fac. Ciencias. Universidad Pais Vasco, \\ Apart. 644, 48080 Bilbao, Spain
}

Infrared multiple photon absorption is known to be observed in some triatomic and most polyatomic molecules. When the number of absorbed photons is high enough the process leads to photodissociation and excess energy is released as vibrational, rotational and translational energy in the electronic ground state of the fragments. The contribution of these energies is determined by standard methods, that include laser induced fluorescence, LIF, and time-resolved Fourier transform spectroscopy, TR-FTS. Examples of selected determinations are shown.

Once produced, identified and characterized, the kinetic behaviour of the transients with selected reactants can be studied. In particular, removal rate constants of substituted carbenes, in specific vibrational and electronic states, by atoms, olefins and simple molecules are mentioned.

KEY WORDS: Spectroscopy, kinetics, transient.

\section{INTRODUCTION}

Infrared multiphoton dissociation, IRMPD, was incorporated in the early 70's to the armoury of preparative methods of transient species in selective states of spectroscopic and kinetic interest. ${ }^{1}$ Other preparative methods including flash photolysis, microwave excitation, UV and visible laser photochemistry, electron and ion bombardment, synchroton radiation, etc. are also commonly used. These methods are in many respects complementary rather than competitive with IRMPD, which has established itself as the most efficient method to create a large variety of diatomic and triatomic transients, including halocarbenes.

IRMPD, besides its scientific interest, has a considerable number of applications, including technical ones. ${ }^{2-4}$ Fields as different as isotopic separation, atmospheric chemistry, astrophysics, combustion, neutral plasmas and semiconductor etching have benefited from studies carried out by or through applications of this technique.

Most experiments of IRMPD are performed by irradiation with efficient and versatile carbon dioxide laser, tunable between 9 and $11 \mu \mathrm{m}$ and able to work in continuous and pulsed mode. ${ }^{5}$ The high fluences (product of intensity and time) required for MPD require the use of pulsed lasers, usually transversely excited

$\dagger$ Author to whom correspondence should be addressed. 
(TEA- $\mathrm{CO}_{2}$ ). The active media of this laser is a mixture of $\mathrm{CO}_{2}, \mathrm{~N}_{2}$ and $\mathrm{He}$ that following an electrical discharge inverts the population of some $\mathrm{CO}_{2}$ vibrational levels. Observed laser emission correspond to the $(100) \leftarrow(001)$ at $10.6 \mathrm{~mm}$ and the $(020) \leftarrow(001)$ at $9.6 \mathrm{~mm}$ transitions. Technical uses of $\mathrm{CO}_{2}$ lasers in material processing led to improving their reliability and performance. However, simple multiline lasers of $15 \mathrm{~J} /$ pulse, that reduce to $8 \mathrm{~J} /$ pulse when working in single line, have enough energy to lead to MPD in most molecules.

Pulses of TEA-CO $\mathrm{CO}_{2}$ lasers, when seen with a nanosecond detector, have a large spike of $\sim 150 \mathrm{~ns}$ of duration followed by a long tail of up to $3 \mu \mathrm{s}$, where one third of the total energy is stored. This shape is far more complicated when seen on a picosecond scale, where the large spike is composed of shorter sharp spikes. ${ }^{6}$ To diminish the influence of undesired shape several improvements has been suggested, among which the following are worthy of mention: optical breakdown, optical delay and amplification of a $Q$-switch selected section of a cw pulse.

In the absorption of high intensity spiked infrared pulses by molecules, the interaction is not only due to their intrinsic features (p.A and $\left.\mathrm{A}^{2}\right)^{7}$ but also by the radiation fluctuations, and statistical theories are expected to describe reasonably well the process. The accepted model ${ }^{8,2-4}$ envisages absorption taking place through three successive regions. At low levels of excitation (region I), absorption is non-resonant owing to the anharmonicity of the normal coordinate potential energy and compensating mechanisms must be in operation. This is commonly considered as the process bottleneck. Once a few photons are absorbed the energy is transferred to the quasicontinuum levels of the whole molecule (region II), where the density of states is high and resonant absorption can always take place at the monochromatic laser frequency. The degree of randomization of internal energy initiated in the quasicontinuum is increased in the continuum above of the dissociation limit, so that statistical theories of molecular decomposition can be used to estimate the distribution of available energy within the products.

A typical experimental setup to carry out the kinetic and spectroscopic measurements is schematically shown in Figure $1 .{ }^{9}$ The system works as follows: $\mathrm{A} \mathrm{CO}_{2}$ laser pulse of known fluence and shape is sent to the vacuum chamber where an appropriate mixture of precursor and argon has been introduced. Absorption of high intensity infrared radiation leads to precursor photodissociation and the fragments are probed with a dye laser coupled to a $15 \mathrm{~ns}$ pulsed Nd:YAG laser in a perpendicular configuration. The emitted light from the excited photofragments is selected with a monochromator, captured with a photomultiplier and routed through an oscilloscope or boxcar integrator to a PC-computer where data are fitted to appropriate models and delivered as hard copy.

A considerable number of triatomic, diatomic and other polyatomic transients have been prepared in selective states, mainly for kinetic and in some cases new spectroscopic studies ${ }^{10}\left(\mathrm{CD}_{2}\right)$. Table 1 collects a few of these transients where IRMPD have some advantage in their study.

Of interest in the understanding the nature of the IRMPD process itself is the determination of the product state distribution of the nascent fragments. Vector properties are not observed because of the statistical nature of the process. 


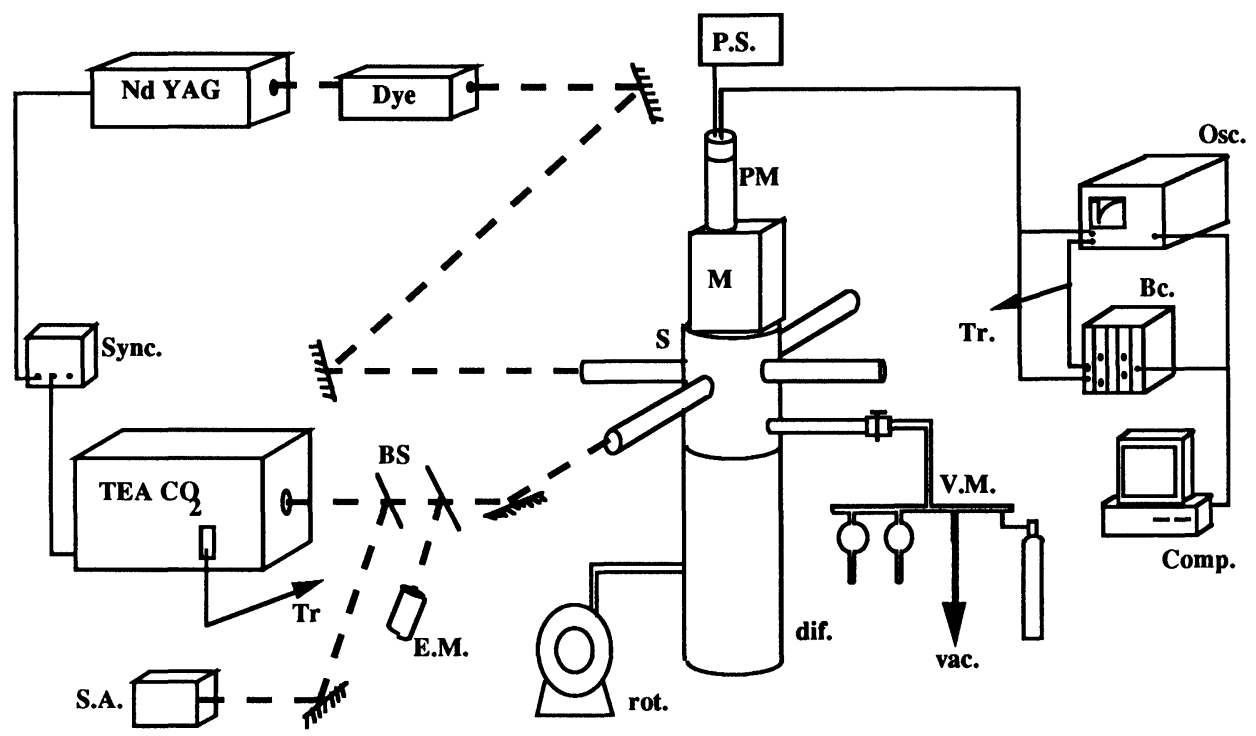

Figure 1 Experimental setup to study the product state distributions and the removal of IRMPD produced free radicals. The following abbreviations are used: Nd: YAG: pulsed Nd:YAG laser. Bc: Boxcar integrator. BS: Beam splitter. Comp: Personal computer, plotter and printer. Dye: Tunable dye laser. EM: Energy Monitor. M: Monochromator/Filter (interference or cut off). Osc: Digital Oscilloscope. PM: photomultiplier. S: Sample space. SA: $\left(\mathrm{CO}_{2}\right.$ laser $)$ spectrum analyzer. SYNC: Electronic laser synchronization. TEA $\mathrm{CO}_{2}$ : Transversely excited $\mathrm{CO}_{2}$ laser. Tr: Trigger. VAC: Vacuum system. VM: Vacuum manifold.

Rotational energy, or its equivalent in a Boltzmann distribution, the rotational temperature, is determined as the best fit of temperature simulated spectra to the experimental one. As rotational relaxation is very fast, the spectra should be measured as close as possible to the laser pulse. As a reference, IRMPD of precursor $\mathrm{CH}_{2} \mathrm{~F}_{2}$ at $0.4,3$ and $10 \mu$ s yield temperatures of 1000,600 and $300 \mathrm{~K}$ respectively. ${ }^{9}$

Determination of vibrational energies requires the knowledge of Franck-Condon factors between vibrational states of the electronic states probed. An alternative method $^{9,11}$ compares the LIF intensities of two sets of three states in the transient species; the excited electronic state is identical in both sets and change of the origin of excitation and the final emission state of the two vibrational states in the electronic ground state gives the ratio of their populations and, therefore, its Boltzmann temperature.

Table 1 Selected transients generated by IRMPD

\begin{tabular}{ll}
\hline Transient & Selected precursor \\
\hline $\mathrm{CHF}\left(\tilde{\mathrm{X}}^{1} \mathrm{~A}^{\prime}\right)$ & $\mathrm{CH}_{2} \mathrm{~F}_{2}, \mathrm{CH}_{2} \mathrm{FCl}$ \\
$\mathrm{CF}_{2}\left(\tilde{\mathrm{X}}^{1} \mathrm{~A}^{\prime}\right)$ & $\mathrm{CF}_{2} \mathrm{Cl}_{2}, \mathrm{CF}_{2} \mathrm{Br}{ }_{2}, \mathrm{CF}_{2} \mathrm{HCl}$ \\
$\mathrm{CFCl}(\tilde{\mathrm{X}})$ & $\mathrm{F}_{2} \mathrm{C}=\mathrm{CFCl}, \mathrm{CFCl}=\mathrm{CFCl}$ \\
$\mathrm{CN}$ & $\mathrm{MeOH}, \mathrm{MeNH}, \mathrm{C}_{2} \mathrm{H}_{4}, \mathrm{EtOH}$ \\
\hline
\end{tabular}


The LIF technique can also be applied to measure the translational energy and the diffusion coefficient by using the so called transient migration method. ${ }^{4,12,13}$ If, for example, the photodissociation and probe laser have a counterpropagating symmetry, the solution of the diffusion equation for very low pressures and assuming a Boltzmann distribution of translational velocities, Yields the time dependent intensity

$$
I(t)=I_{0}\left(R^{2}+L^{2}\right) \cdot\left(R^{2}+L^{2}+c^{2} t^{2}\right)^{-1}
$$

where $R, L$ and $c(=2 \mathrm{kT} / \mathrm{m})^{1 / 2}$ are the photodissociation and probe laser radii and the most probable velocity respectively. For moderate pressures ( $1 \mathrm{~m}$ Torr to 5 Torr) the equation is

$$
I(t)=I_{0}\left(R^{2}+L^{2}\right) \cdot\left(R^{2}+L^{2}+4 D t\right)^{-1}
$$

where $D$ is the diffusion coefficient is obtained. Appropriate fits of $I^{-1}(t)$ versus time squared and time yields the translational temperature and diffusion coefficient. Table 2 collects some state distributions of transients obtained by IRMPD.

Table 2 IRMPD product state distribution for selected halocarbenes and precursors ${ }^{2,3,9}$

\begin{tabular}{lllrr}
\hline Transient & Precursor & $T_{T} / K$ & $T_{R} / K$ & $T_{v} / K$ \\
\hline $\mathrm{CF}_{2}$ & $\mathrm{CF}_{2} \mathrm{Cl}_{2}$ & 510 & 550 & 1050 \\
& $\mathrm{CF}_{2} \mathrm{Br}_{2}$ & 570 & 450 & 790 \\
$\mathrm{CFCl}$ & $\mathrm{CF}_{2} \mathrm{CClF}$ & 370 & $>400$ & 1550 \\
$\mathrm{CHF}$ & $\mathrm{CH}_{2} \mathrm{~F}_{2}$ & 250 & $1000^{\mathrm{a}}$ & $740^{\mathrm{b}}$ \\
& & & $600^{\mathrm{c}}$ & $610^{\mathrm{d}}$ \\
\hline
\end{tabular}

a at $0.4 \mathrm{~ms}$ delay; ${ }^{\mathrm{b}}$ with no $\mathrm{Ar} ;{ }^{\mathrm{c}}$ at $3 \mathrm{~ms}$ delay; ${ }^{\mathrm{d}}$ with 1 Torr of $\mathrm{Ar}$.

The energy distribution and dynamics of diatomic fragments are followed by time resolved Fourier Transform-infrared, tRFT-IR, spectroscopy, set up recently for photodissociation ${ }^{14}$ and kinetic ${ }^{15}$ studies. The advantage of the study of the time evolution of a whole region of the spectra is spectacular.

Once the transient species and their state distributions are identified, kinetic studies may be undertaken with success using gas phase standard techniques. The experimental set up shown in Figure 1 allows the dynamic behaviour of the population of the electronic ground state, the vibrationally excited electronic ground states as well as the electronically accessible states and their vibrational states to be followed.

The rich chemistry of selected states of halocarbenes, including the reactions with atomic species, ${ }^{15}$ the addition to double bonds, ${ }^{9,11}$ the insertion in $\mathrm{CH}$ bonds, the quenching of electro-vibrationally selected species ${ }^{16}$ and chain reactions ${ }^{15,9,17}$ has been recently studied and their constants reported.

\section{References}

1. N. R. Isenor and M. C. Richardson, Appl. Phys. Lett. 18, 224 (1971); Id. Optics Commun. 3, 360 (1971). 
2. M. N. R. Ashfold and G. Hancock, Spec. Period. Reports. Gas Kinetics and Energy Transfer. 3, 73 (1982).

3. D. S. King, Infrared Multiphoton Excitation and Dissociation. Adv. Chem. Phys. L, 105 (1982).

4. D. W. Lupo and M. Quack, Chem. Rev. 87, 181 (1987).

5. A. Yariv, Quantum Electronics. 3rd ed. J. Wiley (1985).

6. R. Naaman and R. N. Zare, Faraday Discuss. Chem. Soc. 67, 242 (1979).

7. H. F. Hameka, Advanced Quantum Chemistry. Addison-Wesley (1965).

8. J. G. Black, E. Tablonovitch N. Bloemberger and S. Mukamel, Phys. Rev. Lett. 38, 131 (1977). T. B. Simpson, J. G. Black, I. Burak, E. Yablonovitch and N. Bloemberger, J. Chem. Phys. 83, 628 (1985). 9. A. Ortiz de Zárate, PhD Thesis. Univ. Pais Vasco, 1991.

10. M. N. R. Ashfold, M. A. Fullstone, G. Hancock and G. W. Ketley. Chem. Phys., 55, 245 (1981).

11. A. Ortiz de Zárate, R. Martinez, M. N. Sanchez Rayo, F. Castaño and G. Hancock. JCS Faraday Trans. (submitted).

12. S. E. Bialkowski, D. S. King and J. C. Stephenson, J. Chem. Phys. 72, 1156 (1980). J. C. Stephenson, S. E. Bialkowski and D. S. King, J. Chem. Phys. 72, 1161 (1980).

13. D. M. Rayner and P. A. Hackett, J. Chem. Phys. 79, 5414 (1983).

14. S. R. Leone, Acc. Chem. Res. 22, 139 (1989) and references therein.

15. G. Hancock and D. E. Heard, JCS. Faraday Trans. 87, 1039, 1045 (1991) and references therein.

16. G. Dornhofer and W. Hack, JCS. Faraday Trans. 11, 84, 441 (1988).

17. G. Hancock and G. Ketley, JCS Faraday Trans. 11, 78, 1283. (1982). 